\title{
Design and Status of the Front Part of the REX-ISOLDE Linac*
}

\author{
T. Sieber ${ }^{a}$ O. Kester ${ }^{a}$ D. Habs ${ }^{\text {a }}$ S. Emhofer ${ }^{a}$ K. Rudolph ${ }^{a}$ \\ H. Bongers ${ }^{a}$ A. Schempp ${ }^{b}$ U. Ratzinger ${ }^{c}$ \\ ${ }^{a}$ Sektion Physik der LMU Muenchen, Am Coulombwall 1, D-85748 Garching \\ ${ }^{\mathrm{b}}$ Institut fuer Angewandte Physik, Robert Mayer-Str. 2-4, D-60325 \\ Frankfurt/Main \\ ${ }^{\mathrm{c}}$ Gesellschaft fuer Schwerionenforschung, D-64220 Darmstadt
}

The accelerator for the REX-ISOLDE experiment at CERN [1] is a compact Linac, which will accelerate neutron rich nuclei with $\mathrm{A} / \mathrm{q}=4.5$ to energies between $0.8 \mathrm{MeV}$ and $2.2 \mathrm{MeV}$ (duty cycle: $10 \%$ ). Substantial components of the REX-ISOLDE Linac, such as the mass separator, the 4-Rod RFQ, the IH-matching section and the IH-drifttube- structure, are developed, built up and tested at Munich. The design and status of these components and the results of beam- and rf-power tests - especially with the RFQ and the IH-structure - are presented in this paper.

\section{Introduction}

REX-ISOLDE is an experiment with energetic radioactive beams at the RIBfacility ISOLDE at CERN [2]. The scheme of REX-ISOLDE is pursued in several steps fulfilling separated functions. Firstly the $1^{+}$ion beam from ISOLDE is accumulated, cooled and bunched in a Penning trap. In an electron beam ion source (EBIS) the $1^{+}$ions are then converted to highly charged ions. In the next step a separator selects the wanted charge-to-mass ratio. This bunched low-energetic beam of highly charged ions is first accelerated in a 4-rod RFQ to $0.3 \mathrm{MeV} / \mathrm{u}$. In the following IH-structure and the three 7-gap resonators the ions are further accelerated to a tunable final energy between $0.8 \mathrm{MeV} / \mathrm{u}$ and $2.2 \mathrm{MeV} / \mathrm{u}$.

\footnotetext{
ऋ work supported by the BMBF under contract 06 LM 868 I/TP:4
} 


\section{The REX-ISOLDE mass separator}

Due to the energy spread of the ions which are expelled from the EBIS $(\Delta \mathrm{E} / \mathrm{E}$ of $\approx 5 * 10^{-3}$ ) a simple magnetic achromat with two $90^{0}$ dipoles is insufficient, because the momentum spread $\Delta \mathrm{p} / \mathrm{p}$ results in a deterioration of the $\frac{\Delta(q / A)}{(q / A)}$ resolution. We therefore employed a separator similar to a Nier-spectrometer [3]. From the known residual gas spectrum of the EBIS it is concluded that a mass separation with a $\mathrm{q} / \mathrm{A}$-resolution of about 1/150 is sufficient to select the highly charged rare radioactive ions with intensities down to $10^{3} / \mathrm{s}$ from rest gas contaminants. The achromatic separator consists of an electrostatic and a magnetic bender. The resolving power of this system depends on the emittance of the injected beam. To provide a resolving power of 150, which is sufficient to separate all isotopes from residual gas ions, the EBIS emittance has to be lower than $10 \pi \mathrm{mm}$ mrad at $20 \mathrm{kV}$ extraction potential.

All components of the separator are delivered to ISOLDE and ready for installation, except for the electrostatic deflector, which is currently completed. The buildup and alignment will start after the emittance measurements at the EBIS.

\section{The REX-RFQ}

The REX-ISOLDE RFQ is a progressive development derived from the GSI HLI-RFQ [4] and from the RFQ of the Heidelberg high-current injector [5]. The RFQ tank is $3 \mathrm{~m}$ long and has a inner diameter of $0.32 \mathrm{~m}$. The modulation of the four electrodes had to be optimized for $\mathrm{A} / \mathrm{q}<4.5$ and for a specific input and output velocity. In order to gain efficient adiabatic bunching and optimum output emittances, we choose a rather low injection energy of 5 $\mathrm{keV} / \mathrm{u}$. The lay-out of the RFQ is conservative and we therefore expect that for the maximum voltages between the rods even charge-to-mass ratios larger than $1 / 6.5$ can be accelerated. This will be important if heavier ions will be used in future experiments.

For the design of the REX-RFQ the assumed normalized injection emittances have been $\epsilon_{x, y}=0.6 \pi \mathrm{mm}$ mrad. The final transverse emittances are $\epsilon_{x, x^{\prime}}=$ $0.68 \pi \mathrm{mm} \operatorname{mrad}$ and $\epsilon_{y, y^{\prime}}=0.67 \pi \mathrm{mm} \operatorname{mrad}$ assuming $95 \%$ transmission. The resulting emittance growth is only $11 \%$. In longitudinal direction the phase spread and the energy spread are $\Delta \varphi= \pm 14^{0}$ and $\Delta W / W= \pm 1.5 \%$.

Low level frequency tuning and flatness measurements resulted in an unflatness of the voltage distribution along the rods below 1.5\%, an $\mathrm{Rp}$ value of 145 $\mathrm{k} \Omega \mathrm{m}$ and a quality factor of 4050 . The power tests have shown a rather short time for conditioning up to $80 \mathrm{~kW}$ and a very good rf-coupling (reflection:$35 \mathrm{~dB}$ ). The Rp-value of $145 \mathrm{k} \Omega \mathrm{m}$ could be verified by X-ray spectroscopy and 

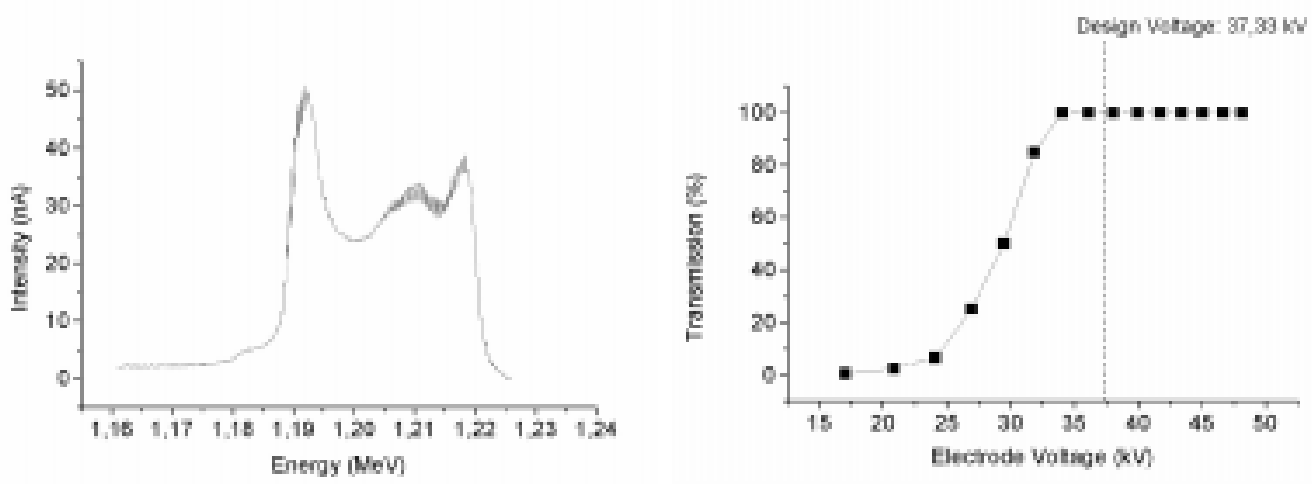

Fig. 1. left: Energy Peak of the REX-RFQ at $28.35 \mathrm{~kW}$ rf-power (electrode voltage: $37.3 \mathrm{kV}$ ). right: Transmission at different electrode voltages.

beam measurements. During the beam tests with $\mathrm{He}^{1+}$, the specifications of a transmission close to $100 \%$ and an energy spread of $\pm 1.5 \%$ could be fulfilled (see Fig. 1).

\section{The matching section between RFQ and IH-structure}

The very special beam dynamics concept of the IH-structure requires a rather small phase spread of the ion bunches and a converging beam in both transverse directions at the entrance of the IH-resonator. For the matching of the beam from the RFQ into the acceptances of the IH-structure, a section consisting of two magnetic quadrupole triplet lenses and a rebuncher has been designed. The rebuncher is a three gap split ring resonator with a peak voltage of $30 \mathrm{kV}$, which has to match the phase spread of $\pm 15^{\circ}$ to the phase spread acceptance of $\pm 10^{\circ}$ of the IH-structure. Measurements at the power resonator resulted in a quality factor of 3700 and a shunt impedance of $11.9 \mathrm{M} \Omega / \mathrm{m}$. In order to reach the peak voltage, $0.3 \mathrm{~kW}$ rf-power will be required. The matching section is currenty in operation at the test beam line at the Garching accelerator laboratory and will be delivered (together with the RFQ) to ISOLDE after bunch- and emittance- measurements behind the rebuncher.

\section{The IH-structure}

The Interdigital-H-type (IH)-structure is an efficient drift-tube structure with special beam dynamics (Combined Zero Degree Structure, KONUS [7]). The IH-structure of REX-ISOLDE is a short version $(1.5 \mathrm{~m}, 20$ gaps) of similar structures like e.g. 'tank 1' of the lead LINAC at CERN [6]. A new feature 
of this accelerator is the possibility to vary the final energy by modification of the gap-voltage distribution via capacitive plungers. The maximum energy gain of about $0.9 \mathrm{MeV} / \mathrm{u}$ corresponds to $4.05 \mathrm{MV}$ effective resonator voltage or 5.04 MV absolute voltage. Assuming a shunt impedance of $300 \mathrm{M} \Omega / \mathrm{m}$, the total peak power consumption will be $60.5 \mathrm{~kW}$.
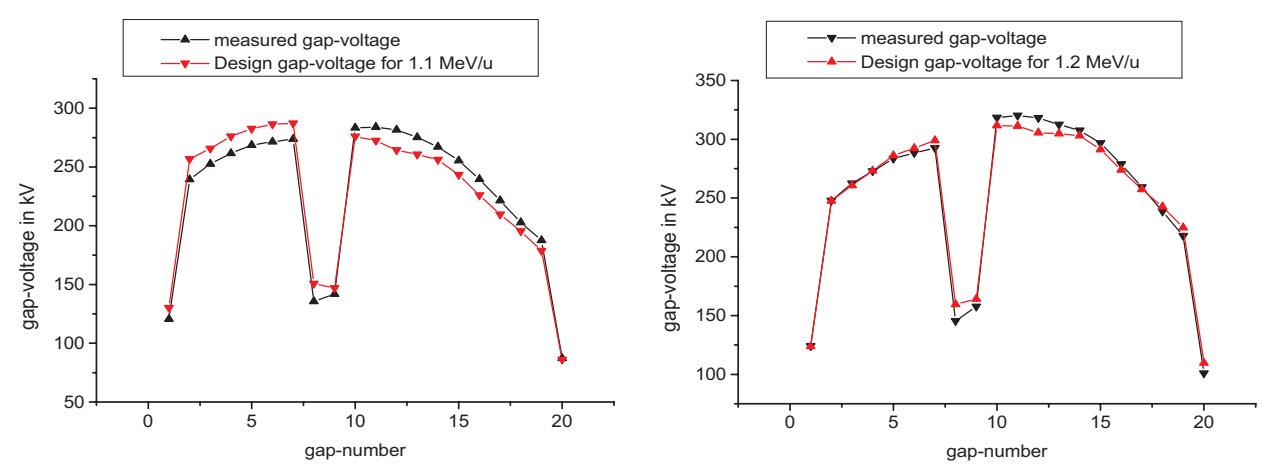

Fig. 2. Design voltage distributions for 1.1 and $1.2 \mathrm{MeV} / \mathrm{u}$ in comparison to the currently measured distributions at $101.28 \mathrm{MHz}$.

A comparison between the reference gap voltage distribution given by particle dynamics calculations and the measured distributions at the model and the power resonator have shown significant discrepancies to calculations with MAFIA within $2 \%$. Thus for the power resonator slight changes of the undercut geometry and of the drift tube structure have to be done in order to increase the resonator frequency which is currently to low for operation in the $1.1 \mathrm{MeV} / \mathrm{u}$ mode (see Fig. 2). The low level measurements of the resonator parameters in the $1.2 \mathrm{MeV}$ mode showed a shunt impedance of $330 \mathrm{M} \Omega / \mathrm{m}$ and a quality factor of 16500 . During the first phase of operation at REX-ISOLDE, the IH-structure can cover an energy range of 1.15 to $1.2 \mathrm{MeV} / \mathrm{u}$.

\section{References}

[1] D. Habs et al., Nucl. Phys. A616 (1997) 29c.

[2] B. Jonson et al., Nucl. Phys. News 3 (1993) 5.

[3] R. Rao, D. Habs, O. Kester, K. Rudolph and T. Sieber in: Proc. of the EPAC98, Stockholm, June 22-26, 1998.

[4] J. Friedrich et al. in: Proceedings of the IEEE PAC 1991, p.3044.

[5] C.-M. Kleffner et al. in: Proceedings of the EPAC 1992, p.1340.

[6] D. Warner et al., "CERN Heavy-Ion Facility Design Report", CERN 93-01 (1993)

[7] U. Ratzinger, Il Nuovo Cimento 106A (1993) 1583 